\title{
A WHOLE BLOOD OXIMETER
}

\author{
BY
}

\section{C. RODDIE,* J. T. SHEPHERD AND R. F. WHELAN}

From the Department of Physiology, The Queen's University of Belfast

Received February 12, 1957

During cardiac catheterization it is an advantage to have an immediate indication of the oxygen content of blood samples withdrawn from the heart and great vessels while the catheter is still in situ. This is especially important in the more complex congenital defects as the procedure can then be modified if necessary in the light of the blood oxygen estimations.

The present paper describes a whole blood cuvette oximeter that fulfils these requirements and employs a standard portable colorimeter.

\section{METHODS}

The Colorimeter. An EEL portable colorimeter is used (Evan's Electroselenium Ltd.). The only modification is that the 2-volt light source is replaced by a 12-volt car bulb powered by a lead accumulator or a constant voltage transformer. The light from this bulb is focused on the cuvette by a convex lens.

The Cuvette. This is made of pyrex glass and consists of a flat chamber with inlet and outlet tubes of about $1 \mathrm{~mm}$. internal diameter (Fig. 1). The depth of the chamber is about $0.2 \mathrm{~mm}$. and $0.2 \mathrm{ml}$. blood is sufficient to fill it and $0.4 \mathrm{ml}$. both the chamber and inlet tubing. The cuvette is mounted with plasticene in a cylindrical brass holder made to the same external dimensions as the standard tubes supplied with the EEL colorimeter. The cuvette is filled through the tube that enters at the bottom since in this way air bubbles are most easily expelled. As the cuvette is not removed from the instrument, the optical properties are constant and it is not necessary that the walls of the cuvette be absolutely flat.

Technique. No filter is employed to reduce the band width of the incident light. An opaque glass filter is constructed so that when it is placed in the light path in front of the cuvette filled with saline the optical density is similar to that of the cuvette filled with blood. This filter provides a reference optical density in setting up the instrument and the optical densities of all blood samples are determined in relation to this reference filter. The optical density of fully reduced and fully oxygenated blood is determined at the commencement of the procedure. For the oxygenated sample, a few $\mathrm{ml}$. of blood are rotated in a small corked flask. To reduce the blood fully $0.1 \mathrm{ml}$. of freshly prepared saturated sodium hydrosulphite solution is added to $5 \mathrm{ml}$. of blood. The test samples are then run through as required and their optical densities determined. The percentage saturation of a given sample is derived from the ratio $D_{R}-D_{T}$ / $D_{R}-D_{0}$, where $D_{R}, D_{o}$ and $D_{T}$ represent the optical densities of the fully

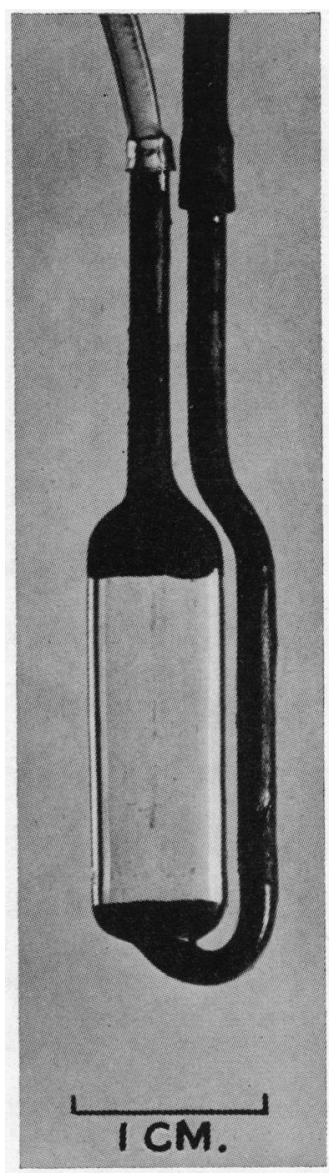

Fig. 1.-The cuvette (see text).

* Walter Dixon Memorial Scholar (British Medical Association). 
reduced, the fully oxygenated, and the test samples respectively. The value so obtained is converted into percentage saturation using the calibration curve shown in Fig. 2.

The oxygen capacity of the blood is derived from the difference in optical density between the fully reduced and the fully oxygenated samples, this value being converted into oxygen capacity in volumes per cent by the use of the calibration curve shown in Fig. 3. The oxygen content is derived from the oxygen saturation and capacity.

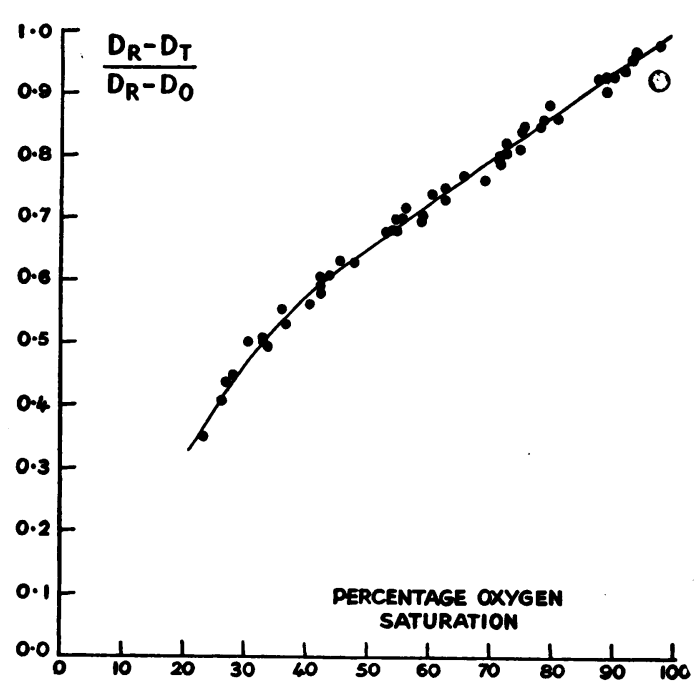

FIG. 2.-Calibration curve for the determination of blood oxygen saturation. $D_{R}, D_{T}$, and $D_{0}$ represent the optical densities of the reduced, test, and oxygenated blood samples respectively.

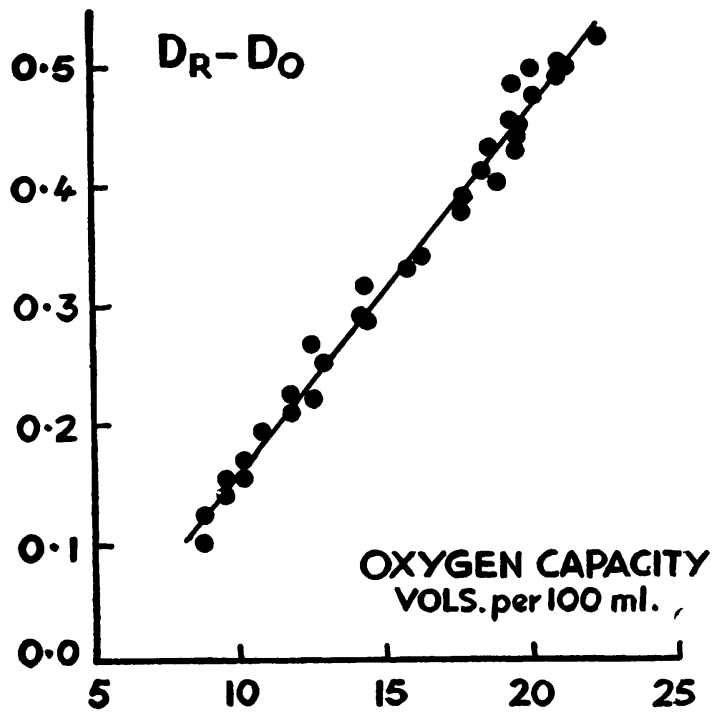

FIG. 3.-Calibration curve for the determination of blood oxygen capacity. $D_{R}$ and $D_{0}$ represent the optical densities of the reduced and oxygenated blood samples respectively.

\section{RESULTS}

The calibration curve from which oxygen saturation is deduced from optical density measurements was constructed by obtaining the oxygen saturation of 50 consecutive blood samples by a spectrophotometric technique employing hæmolysed blood (Roddie et al., 1957) and plotting these values against the optical density measurements. The standard deviation of the individual points from the curve drawn by inspection was 1.74 per cent saturation and this curve (Fig. 2) is used in the subsequent transfer of optical density ratio into percentage oxygen saturation.

The oxygen capacities of 32 blood samples were determined in a similar manner and plotted against the difference in optical densities between reduced and oxygenated samples. The linear regression of the points so obtained was determined and the standard deviation from the regression line was 0.79 volumes per $100 \mathrm{ml}$.

\section{Discussion}

The value of a whole blood cuvette oximeter for cardiac catheterization is undoubted. Wood (1950) has devised a cuvette oximeter with a high relative accuracy, but there is no such instrument commercially available in this country, though Handforth (1952) has devised a cuvette using a modification of the EEL colorimeter which is suitable for whole blood. The present method is based on a spectrophotometer method using hæmolysed blood (Roddie et al., 1957), but as with all photometric methods, calibration curves must be obtained for each cuvette and instrument used. 
From the optical point of view whole blood is an unsatisfactory fluid compared with hæmolysed blood. After the whole blood fills the cuvette the readings do not remain stationary due to changes in orientation and sedimentation of the red blood cells. All the readings must therefore be taken at a standard time after filling the cell. In the technique described in the present paper, the readings were taken immediately after the cell was filled.

\section{SUMMARY}

A simple colorimetric method is described for the rapid estimation of percentage oxygen saturation, content, and capacity of small samples of whole blood. The method employs a small glass cuvette in a slightly modified standard colorimeter which enables $1-\mathrm{ml}$. blood samples to be injected into the cuvette in rapid succession without removal of the cuvette from the instrument.

Calibration curves for percentage oxygen saturation and capacity have been constructed by comparison of the optical density measurements with the values of percentage saturation and capacity obtained by a spectrophotometric technique using hæmolysed blood.

In 50 consecutive blood samples with saturations ranging from 23 to 98 per cent the standard deviation of the differences between values obtained by the two methods was 1.7 per cent. In 32 consecutive blood samples with oxygen capacities ranging from 8.8 to $22 \mathrm{ml}$. per $100 \mathrm{ml}$. the standard deviation of the differences between values obtained by the two methods was 0.79 ml. per $100 \mathrm{ml}$.

We wish to thank Mr. M. L. Speet, Dept. of Physics, The Queen's University of Belfast, who made the cuvettes.

\section{REFERENCES}

Handforth, C. P. (1952). Lancet, 1, 1252.

Roddie, I. C., Shepherd, J. T., and Whelan, R. F. (1957). J. clin. Path., 10, 55.

Wood, E. H. (1950). Medical Physics. Glasser, 2, p. 664 . Year Book Publishers, Chicago. 